\title{
Fertilization strategy and application model using a centrifugal variable-rate fertilizer spreader
}

\author{
Yinyan Shi ${ }^{1}$, Zhichao $\mathrm{Hu}^{2}$, Xiaochan Wang ${ }^{1,3 *}$, Morice O. Odhiambo ${ }^{1}$, Guoxiang Sun ${ }^{1,3}$ \\ (1. College of Engineering, Nanjing Agricultural University, Nanjing 210031, China; \\ 2. Nanjing Research Institute for Agricultural Mechanization, Ministry of Agriculture and Rural Affairs, Nanjing 210014, China; \\ 3. Jiangsu Province Engineering Laboratory for Modern Facilities Agricultural Technology and Equipment, Nanjing 210031, China)
}

\begin{abstract}
With the sustainable development of precision agriculture and the steady progress of variable-rate fertilization technology, the centrifugal variable-rate fertilizer spreader has attracted research attention due to its lower incidence of crush damage, high efficiency, and low cost. To improve fertilization accuracy and uniformity, spreading performance tests were conducted using this spreader in accordance with the test methods specified in ISO 5690 and ASAE S341.2, in which particle mass was weighed in a two-dimensional matrix of collection boxes. The effects of fertilization strategies that control the feed gate flow rate and the disc rotation speed on particle distribution, and application rate per unit area and effective swath width, were investigated. A variable-rate fertilization model was developed by analyzing the variation characteristics resulting from an increasing and decreasing application rate, and field experiments were conducted to verify its accuracy. The results indicated that when the feed gate flow rate was $300 \mathrm{~g} / \mathrm{s}$, the mean application rate was $26.47 \mathrm{~g}$, the standard deviation is 2.81 , and the coefficient of variation of particle distribution is at its minimum value of $14.25 \%$. When the disc rotation speed was $600 \mathrm{r} / \mathrm{min}$, the fertilizer was most evenly distributed with a coefficient of variation of $13.86 \%$, and an average effective spreader swath width of $24.51 \mathrm{~m}$. The proposed variable-rate fertilization model showed a high fitting degree with an S-shaped function curve for both increasing and decreasing distribution rates and the yielding coefficients of determination were more than 0.82 and 0.71 , respectively. The average error between the model predictions and the test results was $9.47 \%$, and the coefficients of determination for the increasing and decreasing distribution rates were 0.91 and 0.82 , respectively, which confirmed the accuracy of the proposed variable-rate fertilization model. This investigation provided a theoretical basis for traditional empirical fertilization using centrifugal variable-rate fertilizer spreaders, and guides the selection of a multiple trajectory, variable-rate fertilization strategy.
\end{abstract}

Keywords: variable-rate fertilization, centrifugal spreader, fertilization strategy, application model

DOI: $10.25165 /$ j.ijabe. 20181106.3789

Citation: Shi Y Y, Hu Z Z, Wang X C, Odhiambo M O, Sun G X. Fertilization strategy and application model using a centrifugal variable-rate fertilizer spreader. Int J Agric \& Biol Eng, 2018; 11(6): 41-48.

\section{Introduction}

With the implementation of China's 'Double Decrease' policy, which calls for the decrease in usage of fertilizer and pesticide, methods for the reasonable application of fertilizer are gaining increased attention in academia, which promotes the development of variable-rate fertilization technology $y^{[1,2]}$. Variable-rate fertilization technology makes some adjustments to traditional balanced fertilization, improves the operational efficiency and utilization rate of fertilizers, and accelerates the sustainable development of modern agriculture to promote high-yield, superior-quality production while ensuring sufficient

Received date: 2017-09-07 Accepted date: 2018-06-19

Biographies: Yinyan Shi, PhD, research interests: agricultural mechanization and automation, agricultural biological environment and energy engineering, Email: 2015212011@njau.edu.cn; Zhichao Hu, Professor, research interests: agricultural electrification and automation, Email: nfzhongzi@163.com; Morice O. Odhiambo, Master, research interests: agricultural biological environment and energy engineering, Email: 1203367199@qq.com; Guoxiang Sun, PhD, Professor, research interests: mechanical and electrical integration, Email: 2013112034@njau.edu.cn.

*Corresponding author: Xiaochan Wang, $\mathrm{PhD}$, Professor, Dean, research interests: agricultural biological environment simulation and control. Department of Electrical Engineering, College of Engineering, Nanjing Agricultural University, Box 96, 40 Dianjiangtai Road, Pukou, Nanjing. Tel: +86- 2558606567, Email: wangxiaochan@njau.edu.cn. environmental protection ${ }^{[3,4]}$. This research discusses a centrifugal variable-rate fertilizer spreader with double conical discs that is well suited to this developmental environment because of its simple structure, high efficiency, and low cost. Therefore, studies worldwide are focusing on the performance of this type of spreader $^{[5,6]}$.

Foreign researchers have been conducting systematic theoretical and experimental analyses of centrifugal fertilizer applicators from the 1980s, when the kinetic characteristics of granular fertilizer on the spreader disc and in the air were first discussed $^{[7-10]}$. Liedekerke et al. ${ }^{[11]}$, van Liedekerke, et al. ${ }^{[12-13]}$ and Coetzee et al. ${ }^{[14]}$ built a simulation model of a centrifugal variable-rate spreader using the EDEM discrete element software, with which the trajectory and dynamic model of fertilizer particles were simulated, and the influence of particle physical parameters and applicator structure parameters on fertilizer distribution were analyzed in detail. Kweon and Grift ${ }^{[15]}$, Fulton et al. ${ }^{[16]}$ and Campbell et al. ${ }^{[17]}$ performed comparison tests using variable-rate fertilizer spreaders consisting of different structures and different control systems (both open loop and closed loop) to determine the effect of these parameters on the performance of the applicator, as well as to establish the variation of fertilizer particle distribution under different influence factors. Recently, Chinese scholars reported numerous successful centrifugal variable-rate fertilization equipment with conical discs ${ }^{[18-20]}$. Chen et al. ${ }^{[21]}$ developed a 
self-propelled, high-ground-clearance spreader for the variable-rate fertilization of rice paddies, in which the machine structural parameters, control system, and general drive chassis were investigated, and a test analysis was conducted to verify its performance. $\mathrm{Hu}$ et al. $^{[22]}$ addressing the particular narrow fertilizing requirements for tea trees, designed a fertilizer spreader with adjustable vanes and an offset centrifugal plate, determined its working parameters, and conducted a regression analysis and parameter optimization based on the discrete element simulation of the spreading process, then experimentally verified the accuracy of the simulation model. Existing research mainly focuses on the optimization of the structural design of the fertilizer applicator and the response of its control system, or on the simulation test analysis of particle movement trajectories and distribution using discrete element software. However, field investigations of spreading strategies and fertilization models using centrifugal variable-rate fertilizer spreaders with conical discs have rarely been reported.

Therefore, in this study, we used the precision variable-rate fertilizer applicator previously developed by our research group for winter wheat based on the canopy spectral reflectance ${ }^{[23]}$, and attempted to address problems related to large application areas, low fertilizer utilization, uneven distribution, etc. We performed spreading performance tests using the developed centrifugal variable-rate fertilizer spreader based on a spectral sensor, evaluated the test results, developed a fertilization model, and verified the model accuracy to improve the operational efficiency and utilization rate of the fertilizer and guide the selection of a multiple-trajectory, multiple-region, and variable-rate fertilization strategy.

\section{Structure and working principle of the fertilizer spreader}

\subsection{Spreader structure}

Figure 1 shows the integral structure of the developed real-time variable-rate fertilizer spreader based on spectrum technology. The spreader consists of a spectral sensor, a vehicle-mounted control terminal (CPU), a walking system, a spreader device, a control system, and other minor parts.

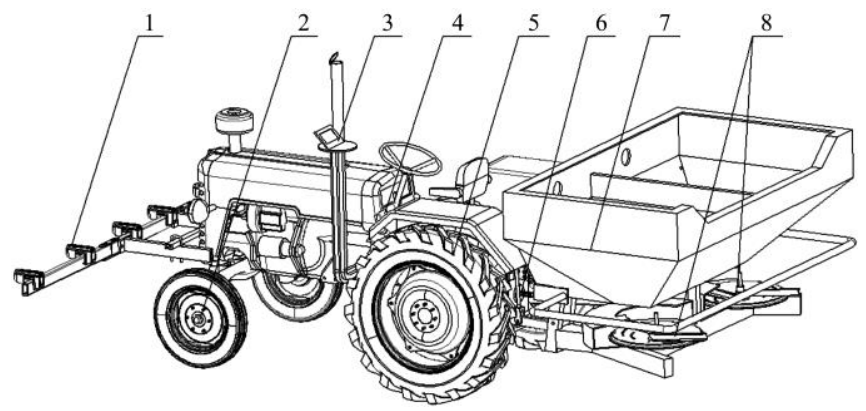

1. Spectral sensor 2. Speed sensor 3. On-board CPU 4. Control system 5. Driving system 6. Transmission system 7. Fertilizer box 8 . Spreader discs Figure 1 Structural diagram of centrifugal variable-rate fertilizer spreader

\subsection{Working principle}

The developed centrifugal variable-rate fertilizer applicator has suspension traction and exhibits a high degree of automation and uniform spreading distribution. These factors improve the fertilizer utilization rate and hence the production efficiency, making the applicator suitable for large-scale modernized agriculture. During the fertilization process, the Normalized Difference Vegetation Index (NDVI) values of the crop canopy are acquired in real time using a Green Seeker ${ }^{\text {TM }}$ spectrum detection system; these values are transmitted to the vehicle-mounted control terminal through a wireless serial interface module. A variable-rate fertilization expert decision system preset into the CPU is run based on the optimization of the Raun model to generate target fertilizer rate in real time. The feedback information of current walking speed, the rotational speed of the disc, and the opening feed gate of fertilizer box (particle flow rate) is integrated. A core controller (STM32 microcontroller) is guided through a decision-making system to drive a stepper motor for adjusting the opening feed gate of fertilizer can. In this manner, online real-time crop fertilizer application is achieved and relative precision variable-rate fertilization is reached.

\section{Materials and methods}

\subsection{Mechanical characteristics of fertilizer}

A commonly used fertilizer based on KCL compound fertilizer was used for the tests in this study. For convenience, it was assumed that fertilizer particles were spherical. The particles were sampled randomly, one hundred particles were selected and their physical properties were determined. The average diameter is $4.02 \mathrm{~mm}$, the spherical rate is more than 0.94 , the average moisture content is $16.42 \%$, the density is $1.38 \times 10^{3} \mathrm{~kg} / \mathrm{m}^{3}$, the hardness is $44.73 \mathrm{~N} / \mathrm{mm}^{2}$, the dynamic friction coefficient is 0.26 , the static friction coefficient is 0.34 , the Poisson's ratio is 0.24 , and the shear modulus is $10.76 \mathrm{MPa}$.

\subsection{Performance test method}

Performance tests were carried out in April 2017 at a test station in Yancheng Yanhai Tractor Manufacturing Co. Ltd. in Jiangsu. The weather was fine, the temperature was $15^{\circ} \mathrm{C}-25^{\circ} \mathrm{C}$, the wind speed was within $2.0 \mathrm{~m} / \mathrm{s}$ (in conformance with the test wind speed stated in the ASAE standard), the ground was relatively flat, the testing area was $450 \mathrm{~m}^{2}$, the relative humidity of air was $42 \%$, and the absolute moisture content of soil was $21 \%$. Test methods and indexes were considered at relevant setting standards according to GB/T5262-2008 (General rules for the determination of agricultural mechanical test conditions), ISO 5690, and ASAE S314.2 $2^{[24,25]}$. The setting of the field experiment is shown in Figure 2.

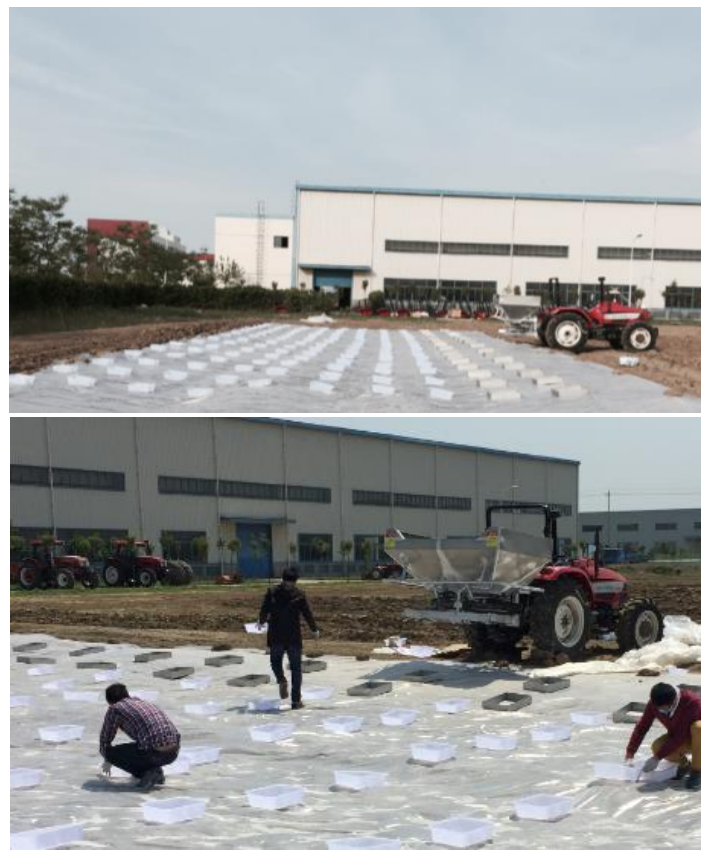

Figure 2 Performance test of spreading fertilizer

The test area $(30 \mathrm{~m} \times 14 \mathrm{~m})$ was covered with plastic film to 
reduce fertilizer wastage, and a moderate amount of soft cotton cloth was pasted in each collection pan for reducing the error caused by fertilizer bounce. One hundred and fifty entity collection pans (each with a size of $50.8 \mathrm{~cm} \times 40.6 \mathrm{~cm} \times 10.2 \mathrm{~cm}$ ) were arranged in 15 rows and 10 columns at intervals of $1.5 \mathrm{~m}$ in the columns and $0.8 \mathrm{~m}$ in the rows. The mass of the fertilizer granules (labeled and saved in bags) in each collection pan was evaluated for the characterization of the transverse distribution of fertilizer particles where the fertilizer spreader passed over the center of a test area at a ground speed of $1.2 \mathrm{~m} / \mathrm{s}$. The fertilizer bulk was adjusted to be approximately $50 \%$ of the total capacity of the can and the spreader was calibrated according to the corresponding working condition for each test. After running steadily in the stable region, the spreader was run in the measurement area (area with collection pans). There was a stop area at the end to ensure the accuracy of the test in determination of region. The coefficient of variation across the transverse distribution of particles was then calculated to assess the spreading performance of the centrifugal variable-rate fertilizer applicator and each group of experiments repeated 3 times. The walking track and the array of collection pans are shown in Figure 3 and the equations for the coefficient of variation are as follows:

$$
\left\{\begin{array}{l}
C V=\frac{S D}{\bar{X}} \times 100 \% \\
S D=\left\{\sum_{i=1}^{n}\left[\left(X_{i}-\bar{X}\right)^{2}\right] /(n-1)\right\}^{\frac{1}{2}} \\
\bar{X}=\frac{1}{n} \sum_{i=1}^{n} X_{i} \quad(i=1,2,3, \ldots, n)
\end{array}\right.
$$

where, $C V$ is the coefficient of variation of particle distribution; $S D$ is the standard deviation of fertilizer quantity; $\bar{X}$ is the average quantity of fertilizer particles; $X i$ is the quantity of fertilizer particles in the $i^{\text {th }}$ collection pan $\mathrm{g}$; and $n$ is the total number of collection pans.

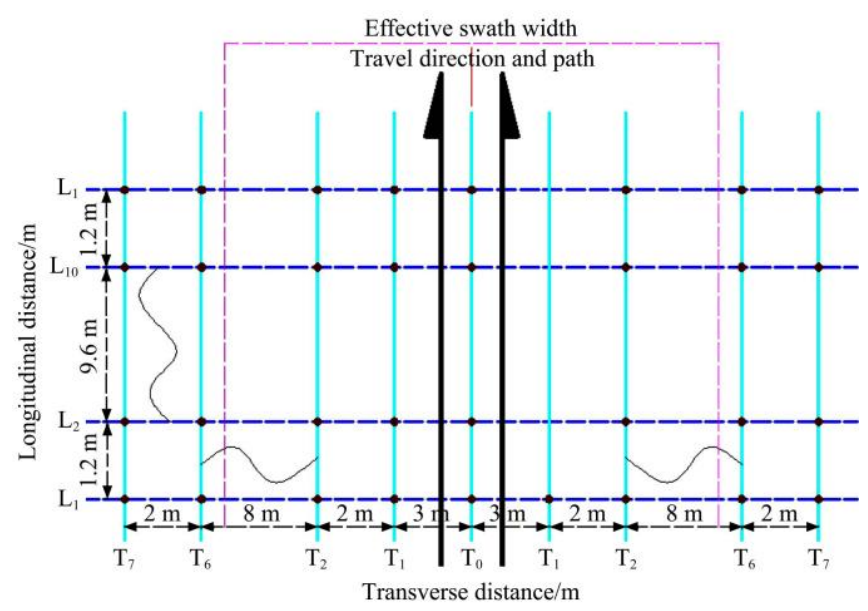

Figure 3 Schematic diagram of collection pan matrix

\subsection{Field test method}

In June 2017, field validation tests were conducted at a rice test base at Dongtai Jinmansui Agricultural Development Co., Ltd. in Jiangsu to verify the spreading performance and prediction accuracy of the variable fertilization model developed in Section 4.2 (for both increasing and decreasing distribution flow rates). The weather was clear, with a temperature between $25^{\circ} \mathrm{C}-36^{\circ} \mathrm{C}$, the wind speed was under $2.0 \mathrm{~m} / \mathrm{s}$, the testing area was 20 acres, the relative humidity of the air was $31 \%$, and the test was conducted in accordance with the methods detailed in Section 3.2.

\section{Results and analysis}

\subsection{Fertilization strategy using feed gate flow rate}

When using the developed variable-rate fertilizer spreader, the application density mainly adjusted by changing the distributor feed gate flow rate. In order to analyze the influence of the distribution flow rate on the spreading characteristics of the centrifugal variable-rate fertilizer applicator, with reference to the current literature ${ }^{[20,22]}$, a disc speed of $\omega=600 \mathrm{r} / \mathrm{min}$, a single-row distribution flow rate range from $q=100 \mathrm{~g} / \mathrm{s}$ to $500 \mathrm{~g} / \mathrm{s}$, and an interval of $100 \mathrm{~g} / \mathrm{s}$, was evaluated. Spreading performance tests for different distribution flow rates within the target range were conducted with the test results shown in Figure 4 and Table 1. It can be seen that the application density increases as the feed gate flow rate increases. The shape of the fertilizer particle distribution gradually changes from a Gaussian-type to an M-type, then to a W-type, and the effective spreading swath width exhibits a slightly upward trend, though this last effect is not significant.

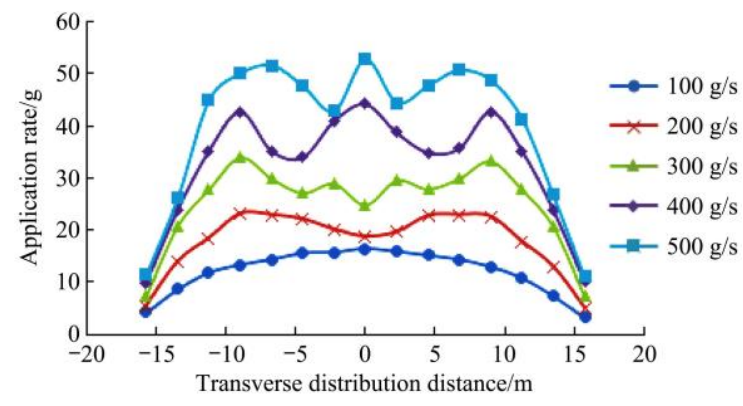

Figure 4 Effects of feed gate flow rate on spreading performance

It can be seen from Table 1 that for a target spreading swath width of $24 \mathrm{~m}$, the coefficient of variation of particle distribution exhibits a rapidly decreasing trend before slowly increasing with the increase in distribution flow rate. When the distribution flow rate $q$ is $100 \mathrm{~g} / \mathrm{s}$, the average fertilizer application rate is $13.23 \mathrm{~g}$, the standard deviation is 1.80 , and the coefficient of variation of particle distribution is at its largest value of $21.65 \%$. When $q=$ $300 \mathrm{~g} / \mathrm{s}$, the average fertilizer application rates is $26.47 \mathrm{~g}$, the standard deviation is 2.81 , the coefficient of variation of particle distribution is at its minimum value of $14.25 \%$. Within the distribution flow rate range of 300-500 g/s, the variation of particle distribution is not significant because the fertilizer application is so small that it results in an irregular and disorderly particle distribution, producing a higher coefficient of variation of particle distribution. When $q=500 \mathrm{~g} / \mathrm{s}$, the average fertilizer application rate is $44.21 \mathrm{~g}$, the standard deviation is 3.70 , and the coefficient of variation of particle distribution is only $19.44 \%$. Further analysis suggests that with the increase in the distribution flow rate, the effective swath width of fertilizer particle distribution increases because as the application rate increases, the interaction force between particles becomes larger. Within the effective spreading swath width, the rate of fertilizer application is slightly higher than the average rate for the entire spreading range: when the distribution flow rate $q$ is $100 \mathrm{~g} / \mathrm{s}$, the average fertilizer application rate is $14.87 \mathrm{~g}$, the coefficient of variation of particle distribution is at its minimum of $10.05 \%$ (for a Gaussian-type advantage), and the coefficient of variation of particle distribution $C v$ for all feed gate flow rates is no greater than $15.26 \%$, which is in compliance with the prescribed requirement that $C v \leq 20 \%$, as specified in NYT1003-2006 'Technical specification of quality evaluation for fertilizing machinery in agricultural industry standard'. 
Table 1 Performance test results of flow rate

\begin{tabular}{|c|c|c|c|c|c|c|c|c|}
\hline \multirow{2}{*}{ Flow rate $/ \mathrm{g} \cdot \mathrm{s}^{-1}$} & \multirow{2}{*}{ Target breadth $/ \mathrm{m}$} & \multicolumn{3}{|c|}{ Application density/g } & \multirow{2}{*}{ Effective breadth/m } & \multicolumn{3}{|c|}{ Application density/g } \\
\hline & & Mean/g & SD & $\mathrm{Cv} / \%$ & & Mean/g & SD & $\mathrm{Cv} / \%$ \\
\hline 100 & & 13.23 & 1.80 & 21.65 & 19.64 & 14.87 & 1.19 & 10.05 \\
\hline 200 & & 19.88 & 2.81 & 17.24 & 21.52 & 20.95 & 1.67 & 12.63 \\
\hline 300 & 24 & 26.47 & 2.63 & 14.25 & 24.06 & 29.19 & 2.84 & 13.89 \\
\hline 400 & & 35.91 & 3.83 & 17.82 & 25.37 & 38.11 & 3.97 & 12.37 \\
\hline 500 & & 44.21 & 3.70 & 19.44 & 27.41 & 47.45 & 3.25 & 15.26 \\
\hline
\end{tabular}

To determine the influence of the distribution flow rate on the spreader performance parameters, regression fittings were conducted between the experimentally set feed gate flow rate and both the measured fertilizer application rate and the effective spreading swath width. The fitting equations and corresponding coefficients of determination are shown in Figure 5 (with $p<0.01$ ). These results indicate that when the distribution flow rate is set within the range of $100-500 \mathrm{~g} / \mathrm{s}$, a quadratic polynomial relationship exists between the application and distribution flow rate, and that there is a linear relationship between the effective swath width and the distribution flow rate. Both relationships exhibit a positive correlation. The fitting models for the application density $\left(y_{\text {rate }}\right)$ and spreading swath width $\left(y_{\text {width }}\right)$ are shown in Equation (2):

$$
\left\{\begin{array}{l}
y_{\text {rate }}=4 E-05 x^{2}+0.0582 x+7.8683 \\
y_{\text {width }}=0.0194 x+17.783
\end{array}\right.
$$

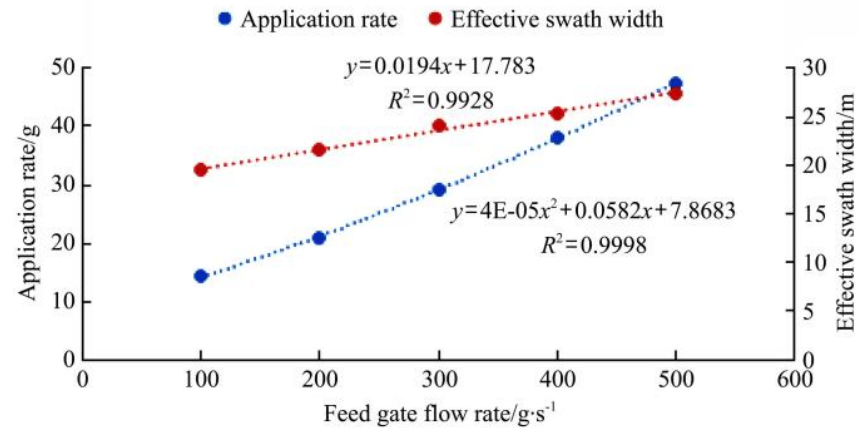

Figure 5 Relationship between feed gate flow rate and application rate, effective swath width

\subsection{Fertilization strategy using disc rotation speed}

The spreader swath width can be adjusted by changing the disc rotation speed in the developed centrifugal variable-rate spreader. The same test methods used to evaluate the effects of changing the feed gate flow rate were employed to study the effect of disc rotation speed on the spreading characteristics of the variable-rate applicator. A distribution flow rate of $q=300 \mathrm{~g} / \mathrm{s}$ was kept constant, and the disc rotation speed was varied from $\omega=300 \mathrm{r} / \mathrm{min}$ to $900 \mathrm{r} / \mathrm{min}$ over intervals of $150 \mathrm{r} / \mathrm{min}$ in order to investigate the influence of disc rotation speed on the spreader performance, with the test results as shown in Figure 6 and Table 2. As the disc rotation speed increases, the effective spreading swath width increases, but the fertilizer application rate in the effective swath width gradually decreases, and the shape of the fertilizer particle distribution gradually shifts from a triangle-type to a W-type, then to an M-type.

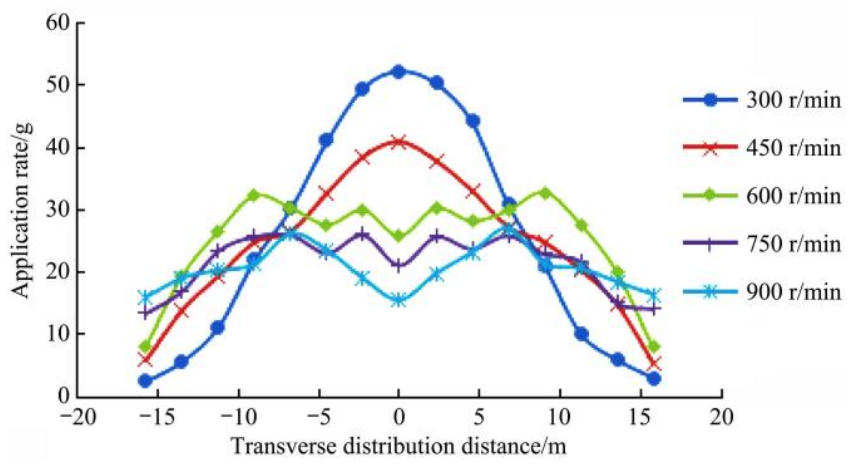

Figure 6 Relationship between disc rotation speed and application rate, effective swath width

Table 2 Performance test results of disc rotation speed

\begin{tabular}{|c|c|c|c|c|c|c|c|c|}
\hline \multirow{2}{*}{ Disc speed $/ \mathrm{r} \cdot \min ^{-1}$} & \multirow{2}{*}{ Effective breadth/m } & \multicolumn{3}{|c|}{ Application density/g } & \multirow{2}{*}{ Target breadth $/ \mathrm{m}$} & \multicolumn{3}{|c|}{ Application density/g } \\
\hline & & Mean/g & SD & $\mathrm{Cv} / \%$ & & Mean/g & SD & $\mathrm{Cv} / \%$ \\
\hline 300 & 12.67 & 42.62 & 9.09 & 21.34 & & 28.80 & 17.45 & 60.58 \\
\hline 450 & 17.84 & 33.24 & 6.26 & 19.66 & & 29.67 & 8.86 & 32.43 \\
\hline 600 & 24.51 & 28.36 & 2.23 & 13.83 & 24 & 27.66 & 4.07 & 14.97 \\
\hline 750 & 25.73 & 26.53 & 3.48 & 15.35 & & 24.15 & 3.48 & 15.19 \\
\hline 900 & 28.09 & 25.18 & 3.16 & 14.42 & & 21.56 & 3.16 & 14.72 \\
\hline
\end{tabular}

From Figure 6 and Table 2, it can be seen that for a target application swath width of $24 \mathrm{~m}$, the coefficient of variation of fertilizer particle distribution initially decreases rapidly, then remains basically stable with the increase in disc rotation speed. When the disc rotation speed $\omega$ is $300 \mathrm{r} / \mathrm{min}$, the average fertilizer application rate is $28.80 \mathrm{~g}$, the standard deviation is 17.45 , and the coefficient of variation of particle distribution is at its maximum of $60.58 \%$. When $\omega=900 \mathrm{r} / \mathrm{min}$, the average fertilizer application rate is $21.56 \mathrm{~g}$, the standard deviation is 3.16 , and the coefficient of variation of particle distribution is at its minimum of $14.72 \%$. Through further analysis, it can be seen that the spreader disc rotation speed has a more significant influence on the effective spreading swath width than on the fertilizer application density. When the disc rotation speed $\omega$ is $300 \mathrm{r} / \mathrm{min}$, the mean effective swath width is at its minimum of $12.67 \mathrm{~m}$, the average fertilizer application rate is $42.62 \mathrm{~g}$, the standard deviation is 9.09 , and the coefficient of variation of particle distribution is at its maximum of $21.34 \%$. When $\omega=900 \mathrm{r} / \mathrm{min}$, the mean effective swath width is at its maximum of $28.09 \mathrm{~m}$, the average fertilizer application rate is $25.18 \mathrm{~g}$, the standard deviation is 3.16 , and the coefficient of variation is $14.42 \%$. Note that the application rate within the effective swath width appears to be generally greater than over the 
entire spreading width: this can be attributed to the fact that as the disc rotation speed increases, the centrifugal force applied to the particles will be large, and the kinematic velocity will increase, which causes the spreader swath width to increase accordingly, resulting in a relatively uniform particle distribution. This has the effect of applying less of the fertilizer within a concentrated area under conditions of the constant distribution flow rate. However, when the disc rotation speed is greater than $600 \mathrm{r} / \mathrm{min}$, the variation pattern of particle distribution is not obvious, the coefficient of variation is no greater than $15.26 \%$, and the effective swath width trends toward approximate stability. When subjected to high rotation speeds, fertilizer particle classification is evident, and the consumed power increases, therefore a $\omega$ of $600 \mathrm{r} / \mathrm{min}$ is identified as the critical rotation speed for spreading, yielding an average effective swath width of $24.51 \mathrm{~m}$, an average fertilizer application rate of $28.36 \mathrm{~g}$, a standard deviation of 2.23 , and a minimal coefficient of variation of particle distribution of $13.83 \%$, indicating the best spreading uniformity.

To quantify the effect of the disc rotation speed on spreader performance parameters, regression fittings were conducted between the disc rotation speed and both the effective spreading swath width and fertilizer application rate. The fitting equations and coefficients of determination are as shown in Figure 7 (with $p<0.05$ ). These results indicate that for a disc rotation speed between 300 and $900 \mathrm{r} / \mathrm{min}$, a positive quadratic polynomial relationship exists between the measured effective swath width and the disc rotation speed, while a negative power correlation exists between the measured application rate and the disc rotation speed. The fitting models for the effective width $\left(y_{\text {width }}\right)$ and application rate $\left(\mathrm{y}_{\text {rate }}\right)$ are as shown in Equation (3).

$$
\left\{\begin{array}{l}
y_{\text {width }}=-4 E-05 x^{2}+0.0725 x-5.994 \\
y_{\text {rate }}=1615.4 x^{-0.638}
\end{array}\right.
$$

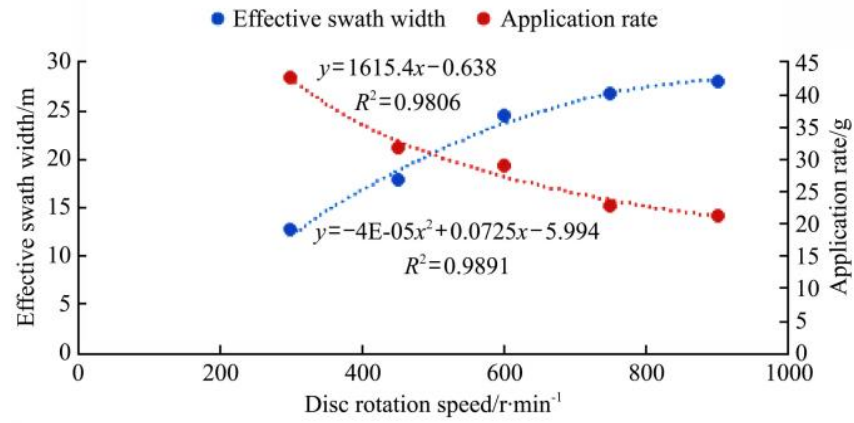

Figure 7 Effects of disc rotation speed on spreading performance

\subsection{Fertilization strategy using variable control}

In the developed centrifugal variable-rate fertilizer spreader, the fertilizer application rate is adjusted by changing the feed gate opening according to the crop growth information acquired by the spectral sensor in real-time. In order to study the fertilizer particle distribution resulting from the process of variable fertilization and to evaluate the variable spreading performance of the developed spreader, the spreader ground speed $v$ was set at $1.2 \mathrm{~m} / \mathrm{s}$, the disc rotation speed $\omega$ was set at $600 \mathrm{r} / \mathrm{min}$, and the distribution flow rate $q$ was continuously varied, increasing from $100 \mathrm{~g} / \mathrm{s}$ up to $500 \mathrm{~g} / \mathrm{s}$, and decreasing from $500 \mathrm{~g} / \mathrm{s}$ down to $100 \mathrm{~g} / \mathrm{s}$. This variable-rate fertilization performance test was implemented, yielding the test results shown in Figure 8. Please note that Figure 8 assumes that the $0 \mathrm{~m}$ point on the longitudinal axis is the starting point of variable-rate fertilization (prior to which the distribution rate is considered to be steady at $q=100 \mathrm{~g} / \mathrm{s}$ ), and the $40 \mathrm{~m}$ point on the longitudinal axis is the end point of variable-rate fertilization (after which the distribution rate is considered to be steady at $q=500 \mathrm{~g} / \mathrm{s}$ ).

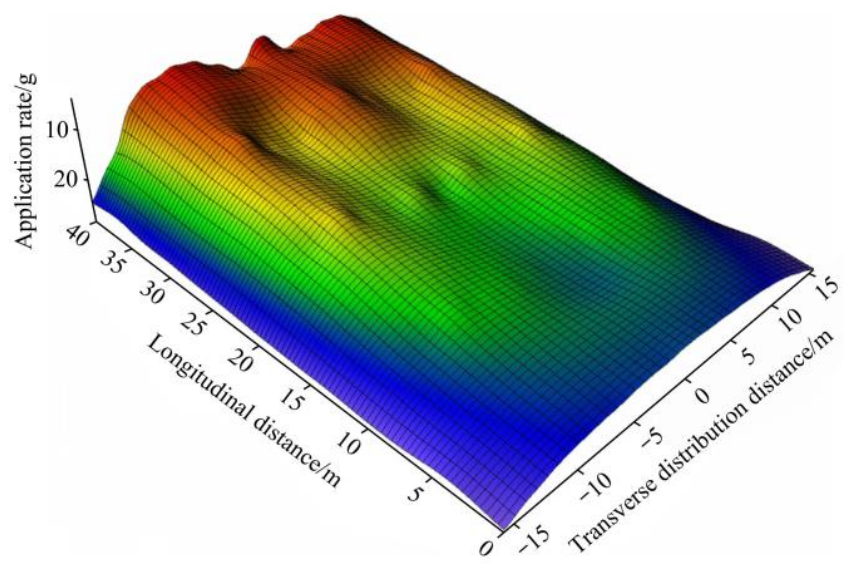

a. Increasing fertilization

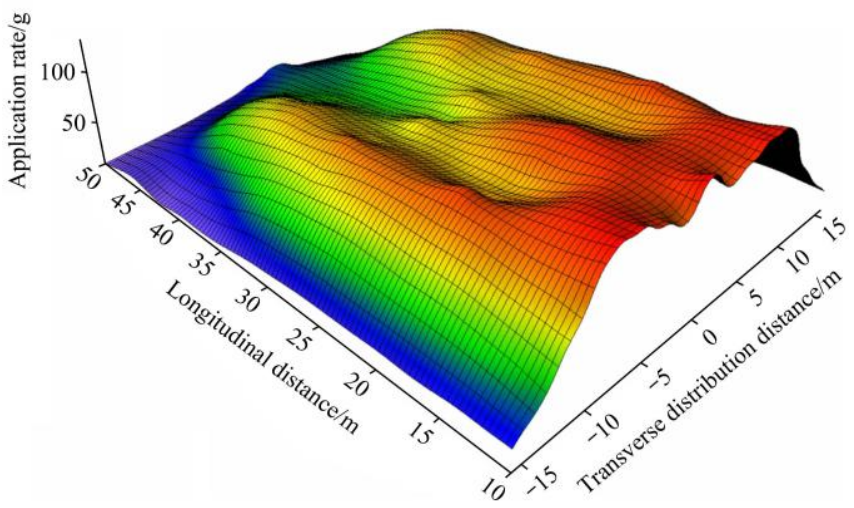

b. Decreasing fertilization

Figure 8 Effects of variable-rate application on spreading performance

It can be seen from Figure 8 that as the distribution flow rate increases from $100 \mathrm{~g} / \mathrm{s}$ to $500 \mathrm{~g} / \mathrm{s}$, the fertilizer particle distribution gradually shifts from a Gaussian-type to an M-type, then to a $\mathrm{W}$-type distribution, which is consistent with the analysis results in Section 4.1. As the distribution flow rate is reduced from $500 \mathrm{~g} / \mathrm{s}$ to $100 \mathrm{~g} / \mathrm{s}$, the particle distribution shape undergoes a similar transformation, but the change characteristics are different; this is related to the distribution actuator control system and the tendency of fertilizer particles to readily distribute. Additionally, during the process of variable fertilization, as the fertilizer application rate increases, the effective spreading swath width increases, meaning that in order to ensure the quality of subsequent multi-trajectory fertilization using a centrifugal variable applicator, the scale of variable rate fertilization should be restricted within the same trajectory to the extent possible, reducing the increment of effective fertilizer swath width and improving the distribution of fertilization within the swath.

To analyze the variation characteristics associated with the application rate of variable fertilization and to improve the fertilizer accuracy of the variable-rate spreader, a regression analysis of the longitudinal distribution of fertilizer particles was conducted. This nonlinear regression fitting was accomplished using an S-type function, establishing mathematical models describing the effects of variable rate fertilization. Based on the structural characteristics of the centrifugal variable-rate fertilizer spreader with double discs, it was assumed that the transverse distribution of fertilizer particles was symmetrical about the spreader walking trajectory $(W=0)$, and a stochastic analysis of particle mass in the 
collection boxes on either side of this center of symmetry center was conducted for each test, with boxes located at a transverse distance from the centerline of $W=0 \mathrm{~m}, \pm 2.25 \mathrm{~m}, \pm 4.5 \mathrm{~m}, \pm 6.75 \mathrm{~m}$, $\pm 9 \mathrm{~m}, \pm 11.25 \mathrm{~m}, \pm 13.55 \mathrm{~m}, \pm 15.75 \mathrm{~m}$, respectively. Each test was repeated three times and the measured data was averaged for analysis. The S-type regression function used is shown in Equation (4) ${ }^{[26]}$, and the regression fitting curves are shown in Figure 9 (where the colored lines represent the collection boxes at each transverse distance).

$$
y=y_{0}+\frac{a}{1+e^{-\left(\frac{x-x_{0}}{b}\right)}}
$$

where, $x$ is the longitudinal distance in $\mathrm{m} ; x_{0}$ is the corresponding longitudinal distance when $y$ is the half of maximum in $\mathrm{m} ; y_{0}$ is the termination application rate (extremum) in $\mathrm{g}$; $a$ is the error between the maximum and minimum values of $y$ (the range) in $\mathrm{g}$; $b$ is a constant.

Table 3 describes the corresponding variable fertilization models as determined using the nonlinear regression fitting curves when the distribution flow rate of the developed spreader continuously increases (from $100 \mathrm{~g} / \mathrm{s}$ to $500 \mathrm{~g} / \mathrm{s}$ ) and decreases (from $500 \mathrm{~g} / \mathrm{s}$ to $100 \mathrm{~g} / \mathrm{s}$ ). When reviewed in conjunction with Figure 9, it can be seen in Table 3 that when the fertilization rate increases, the S-type regression curve has the closest degree of fitting, with $R^{2}=0.99$ at a transverse distribution distance of $W=0$ $\mathrm{m}$ (i.e. at the center of symmetric distribution), indicating that the regression forecasting model of variable fertilization is quite accurate. As the transverse particle distribution extends outward, the fertilizer application rate gradually decreases, and the degree of fitting is relatively lower, though still maintained above 0.82 . When the distribution flow rate decreases, a similar behavior can be observed: at $W=0 \mathrm{~m}$ : the degree of fitting of the regression curve is at its best at $R^{2}=0.86$, and all values of $R^{2}$ are above 0.71 .
Note, however, that the overall fit is less significant than that determined for the increasing distribution flow rate, likely due to the special forming-manner of multiple stacking for application rate using a centrifugal variable-rate fertilizer spreader.

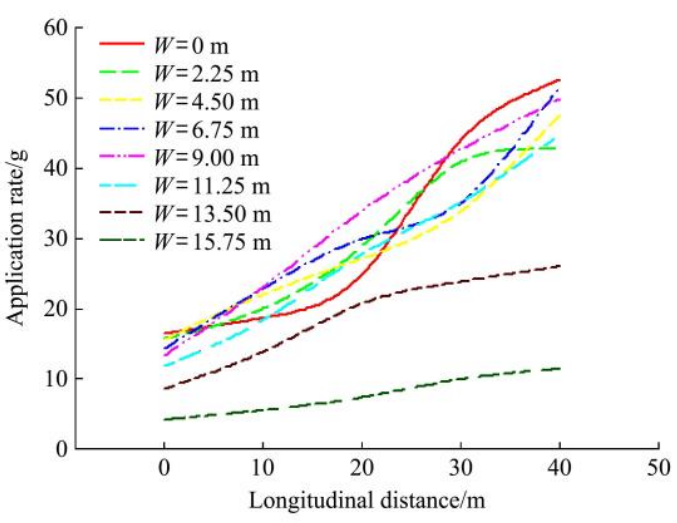

a. Increasing fertilization

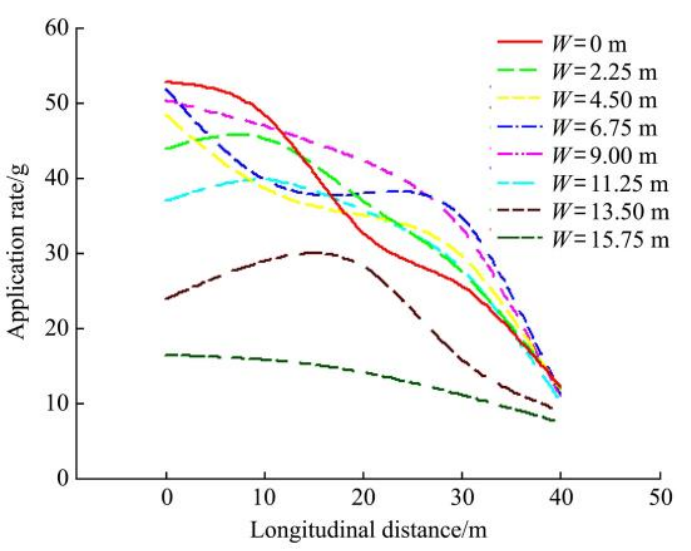

b. Decreasing fertilization

Figure 9 Regression curves of variable-rate fertilization model

Table 3 Parameters of variable-rate fertilization model

\begin{tabular}{|c|c|c|c|c|c|c|c|c|c|}
\hline \multirow{2}{*}{\multicolumn{2}{|c|}{ Parameters }} & \multicolumn{8}{|c|}{ Transverse pan position /m } \\
\hline & & 0 & \pm 2.25 & \pm 4.5 & \pm 6.75 & \pm 9.0 & \pm 11.25 & \pm 13.5 & \pm 15.75 \\
\hline \multirow{5}{*}{ Increasing } & $a$ & 37.18 & 28.16 & 19.87 & 88.53 & 69.91 & 135.78 & 22.19 & 9.37 \\
\hline & $b$ & 4.41 & 5.22 & 5.35 & 3.65 & 16.53 & 37.36 & 8.27 & 9.55 \\
\hline & $x_{0}$ & 25.59 & 20.13 & 24.57 & 34.23 & 12.83 & 40.88 & 12.07 & 22.42 \\
\hline & $y_{0}$ & 16.89 & 15.67 & 18.21 & -12.28 & -8.71 & -22.37 & 4.37 & 3.38 \\
\hline & $R^{2}$ & 0.99 & 0.94 & 0.88 & 0.85 & 0.96 & 0.93 & 0.87 & 0.81 \\
\hline \multirow{5}{*}{ Decreasing } & $a$ & 37.18 & 32.98 & 47.56 & 97.20 & 55.52 & 44.76 & 24.43 & 9.74 \\
\hline & $b$ & -4.41 & -7.56 & -10.42 & -5.69 & -12.60 & -11.88 & -8.34 & -8.84 \\
\hline & $x_{0}$ & 14.40 & 18.98 & 29.68 & 17.41 & 25.36 & 23.00 & 27.50 & 21.59 \\
\hline & $y_{0}$ & 16.89 & 13.75 & 11.36 & 4.15 & -0.25 & 2.08 & 2.80 & 2.20 \\
\hline & $R^{2}$ & 0.86 & 0.83 & 0.72 & 0.80 & 0.84 & 0.75 & 0.81 & 0.71 \\
\hline
\end{tabular}

\subsection{Model validation tests}

The spreading model validation test site is shown in Figure 10. As well, the spreader ground speed $v$ was set at $1.2 \mathrm{~m} / \mathrm{s}$, the disc rotation speed $\omega$ was set at $600 \mathrm{r} / \mathrm{min}$, and the distribution flow rate $q$ was continuously varied, increasing from $100 \mathrm{~g} / \mathrm{s}$ up to $500 \mathrm{~g} / \mathrm{s}$, and decreasing from $500 \mathrm{~g} / \mathrm{s}$ down to $100 \mathrm{~g} / \mathrm{s}$, respectively.

Two types of variable fertilization field validation tests using the developed centrifugal spreader were conducted, evaluating the performance of the derived models under increasing and decreasing distribution flow rates, with each test repeated three times. The average particle mass at each measurement point in the test area was acquired with the results shown in Figure 11. The mean relative fertilizer application rate error between the model forecast and the experimental results is $9.47 \%$, with a maximum error of $16.62 \%$, a minimum error of $4.19 \%$, and a deviation of $4.84 \%$, indicating that the predictive accuracy of the variable-rate fertilization model is above $80 \%$. The correlation of predicted and experimental fertilizer application rate is shown in Figure 12.

The correlation analysis of the variable-rate fertilization model and experimental results in Figure 12 show that the determination coefficient $R^{2}$ for the increasing rate and decreasing rate tests is 0.91 and 0.82 , respectively, and the correlation line slope is 0.98 and 0.87 , respectively. These results suggest that the degree of fitting between the model predictions and the experimental measurements is good, indicating certain accurate predictions and validating the established model for variable fertilization and the 
reference values selected. In all, the field tests confirm that the distribution model for the developed centrifugal variable-rate fertilizer spreader successfully provides certain accurate projection of variable fertilizer application.
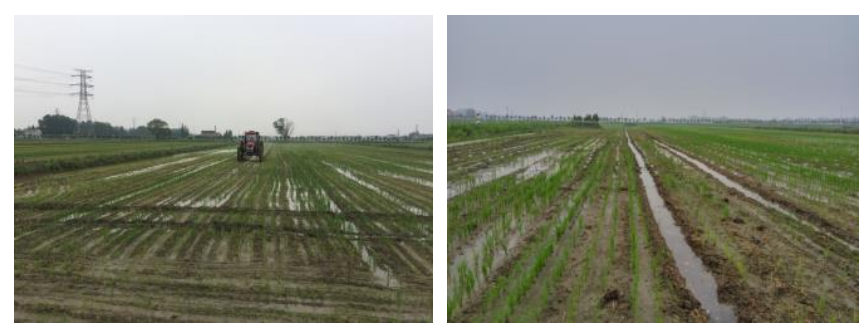

Figure 10 Picture of field validation test

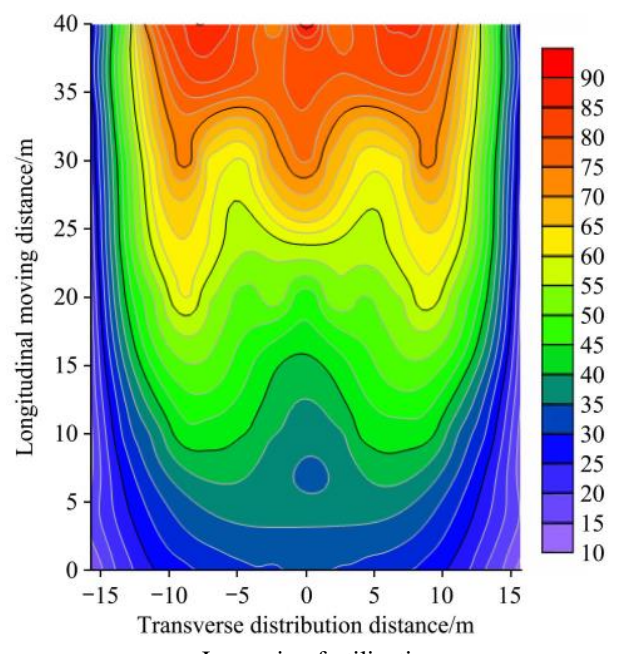

a. Increasing fertilization

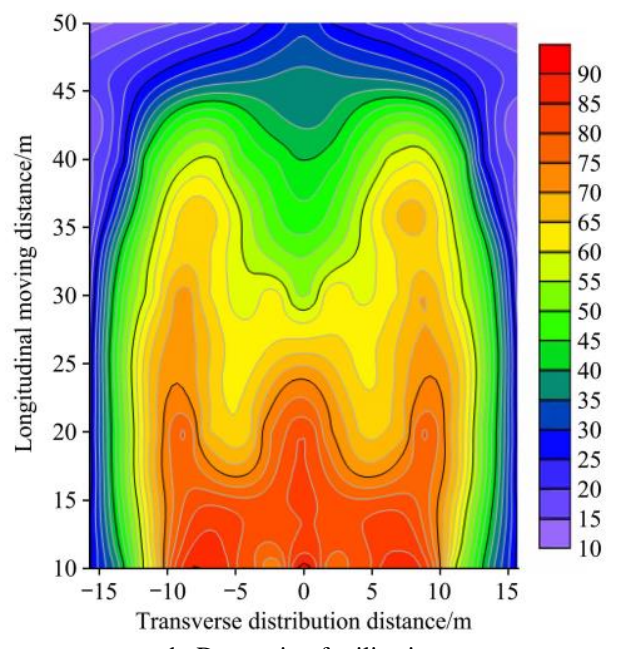

b. Decreasing fertilization

Figure 11 Results of field validation test

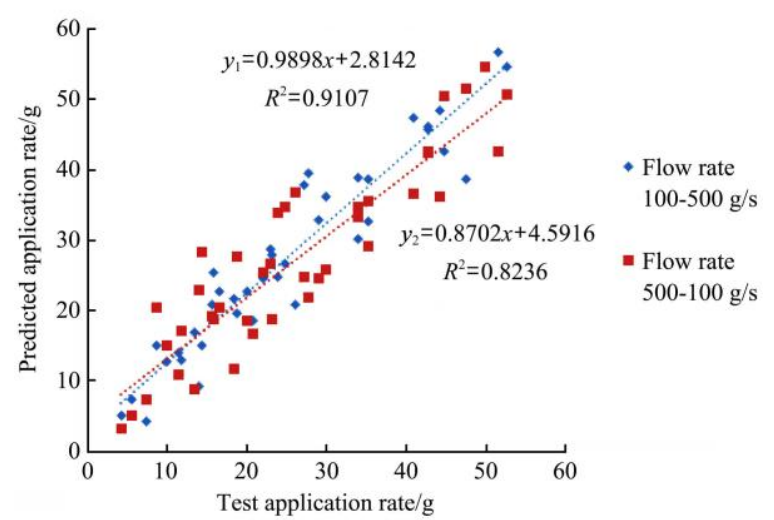

Figure 12 Correlation analysis of test values and predicted values

\section{Discussion}

Recent years, the centrifugal variable-rate fertilizer applicator has obtained an increasing amount of attention, and the traditional fertilizer remains entirely dependent on farmers' empiric fertilization. However, it is impossible to predict the spreader operation swath width and attain accurate fertilizer application rate per unit area only by experience because of the constraints in varying working environments, working parameters, and fertilizer characteristics.

Thus, in this investigation, the importance of selecting fertilizer strategies on the basis of the working environment requirements should be emphasized. For the regional operation block fields, strategies addressing fertilizer application accuracy and distribution uniformity should be prioritized. The results of the current research show that when the distribution flow rate is $100 \mathrm{~g} / \mathrm{s}$, the average fertilizer application rate per unit area is $14.87 \mathrm{~g}$, and the coefficient of variation of particle distribution within the effective fertilization width is at its minimum of $10.05 \%$, indicating high fertilization accuracy. In the case of large rotation planting areas, strategies that prioritize fertilizer efficiency and improve the effective fertilizer swath width should be used. The results of the current research indicate that when the disc rotation speed is $900 \mathrm{r} / \mathrm{min}$, the effective spreader swath width is at its maximum of $28.09 \mathrm{~m}$, the average application rate is $25.18 \mathrm{~g}$, and the coefficient of variation is $14.42 \%$, indicating optimal fertilization efficiency. It is clear from these results that the distribution flow rate (as determined by the feed gate opening) has a significant effect on the fertilizer application density, ensuring accurate fertilizer distribution, while the disc rotation speed mainly influences the effective width of the fertilizer swath, which itself contributes to efficient fertilizer. Therefore, selecting a proper fertilization strategy for field spreading operations is essential to obtaining the best performance from a variable-rate applicator, and consequently realizing the most effective use of fertilizer.

The results of this research can be used to improve the fertilization accuracy and fertilizer utilization of the variable-rate fertilizer applicator, and can help guide a strategy for multi-trajectory, multi-regional variable fertilization. However, the effect of factors such as the control system response speed, fertilizer applicator ground speed, natural environment (i.e. wind speed), etc., on the particle distribution and fertilization accuracy in the field spreading process remain to be addressed in future research, which will provide an academic basis for further study of centrifugal variable-rate fertilization equipment.

\section{Conclusions}

For a fertilization strategy based on the distribution flow rate, the increase in distribution flow rate results in an initially gradual decrease, then a steady increase in the coefficient of variation of particle distribution. When the distribution flow rate is $300 \mathrm{~g} / \mathrm{s}$, the average fertilizer application rate is $26.47 \mathrm{~g}$, the coefficient of variation of particle distribution is at its minimum value of $14.25 \%$, indicating high fertilization accuracy, and there exists a positive quadratic polynomial relationship between the application rate and the distribution flow rate (with $p<0.01$ ).

For a fertilization strategy based on the speed of disc rotation, the effective spreader swath width increases with the increase in disc rotation speed. When the disc rotation speed is $600 \mathrm{r} / \mathrm{min}$ (taken as the critical speed), the effective swath width is $24.51 \mathrm{~m}$ with uniform fertilizer distribution. The coefficient of variation is 
$13.83 \%$, indicating high fertilizer efficiency, and a positive quadratic polynomial function relationship exists between the effective swath width and the disc rotation speed (with $p<0.05$ ).

For a variable-rate fertilization strategy, there exists high degree of fitting between the longitudinal model variation characteristics of fertilizer application rate and an S-type regression function curve. As the flow rate of distribution increases, the degree of fitting for the transverse swath width has a coefficient of determination $\left(R^{2}\right)$ value greater than 0.82 , and as the feed rate of distribution decreases, the degree of fitting has an $R^{2}$ value greater than 0.71 . The fertilizer application density model validation tests produced an average error between the model-predicted value and the experimentally observed value of $9.47 \%$, and the accuracy of the forecast model was above $80 \%$. For the increasing and decreasing distribution flow rates, the coefficients of determination $R^{2}$ were 0.91 and 0.82 , respectively, confirming the validity of the prediction model for variable-rate fertilization, and providing a reference value for traditional empirical fertilization.

\section{Acknowledgements}

The authors acknowledge the financial support provided by National Key Project of Research and Development Program (Grant No. 2016YFD0200600-2016YFD0200602-4) and Projects funded from special funds of the National Modern Agricultural Industry and Technology System (Grant No. CARS-14-Mechanized equipment). The authors would like to thank teacher's and supervisor's technical supports. We also appreciate the assistance provided by brothers and sisters during the tests, and we would like to thank Editage for English language editing. We are grateful for the editors and anonymous reviewers for providing helpful suggestions to improve the quality of the present paper.

\section{[References]}

[1] Ma X L, Fan G B, Li Y Y, Xu Y, Peng Y K. Establishment of precision fertilization decision-making model and database system. Transactions of the CSAM, 2011; 42(5): 193-197. (in Chinese)

[2] Song X Y, Wang J H, Huang W J, Yan G J, Chang H. Monitoring spatial variance of winter wheat growth and grain quality under variable-rate fertilization conditions by remote sensing data. Transactions of the CSAE, 2009; 25(9): 155-162. (in Chinese)

[3] Sui R X, Thomasson J A, Ge Y F. Development of sensor systems for precision agriculture in cotton. Int J Agric \& Biol Eng, 2012; 5(4): 1-14

[4] Chen L P. Theoretical and experimental studies on variable-rate fertilization in precision farming. Beijing: China Agricultural University, 2003: 83-97.

[5] Yuan X, Qi L J, Wang H, Huang S K, Ji R H, Zhang J H. Spraying parameters optimization of swing, automatic variables and greenhouse mist sprayer with response surface method. Transactions of the CSAM, 2012; 43(4): 45-50, 54. (in Chinese)

[6] Wang H, Bai X P, Liang H B. Proportional distribution method for estimating actual grain flow under combine harvester dynamics. Int $\mathbf{J}$ Agric \& Biol Eng, 2017; 10(4): 158-164
[7] Aphale A, Bolander N, Park J, Shaw L, Svec J, Wassgren C. Granular fertiliser particle dynamics on and off a spinner spreader. Biosystems Engineering, 2003; 85(3): 319-329.

[8] Jones J R, Lawrence H G, Yule I J. A statistical comparison of international fertiliser spreader test methods-Confidence in bout width calculations. Powder Technology, 2008; 184(3): 337-351.

[9] Dintwa E, Tijskens E, Olieslagers R, de Baerdemaeker J, Ramon H Calibration of a spinning disc spreader simulation model for accurate dite-specific fertiliser application. Biosystems Engineering, 2004; 88(1): 49-62.

[10] Cool S, Pieters J, Mertens K C, Hijazi B, Vangeyte J. A simulation of the influence of spinning on the ballistic flight of spherical fertiliser grains. Computers \& Electronics in Agriculture, 2014; 105(5): 121-131.

[11] Van Liedekerke P, Tijskens E, Dintwa E, Rioual F. Vangeyte J, Ramon H DEM simulations of the particle flow on a centrifugal fertilizer spreader. Biosystems Engineering, 2009; 190(3): 348-360.

[12] Van Liedekerke P, Tujskens E, Dintwa E, Ramon H. A discrete element model for simulation of a spinning disc fertilizer spreader I. Single particle simulations. Communications in Agricultural \& Applied Biological Sciences, 2006; 170(2): 71-85.

[13] Van Liedekerke P, Tujskens E, Ramon H. Discrete element simulations of the influence of fertiliser physical properties on the spread pattern from spinning disc spreaders. Biosystems Engineering, 2009; 102(4): 392-405.

[14] Coetzee C J, Lombard S G. Discrete element method modelling of a centrifugal fertiliser spreader. Biosystems Engineering, 2011; 109(4): 308-325.

[15] Kweon G, Grift T E. Feed gate sdaptation of a spinner spreader for uniformity control. Biosystems Engineering, 2006; 95(1): 19-34.

[16] Fulton J P, Shearer S A, Higgins S F, Hancock D W, Stombaugh T S Distribution pattern variability of granular VRT applicators. Transactions of the ASAE, 2005; 48(6): 2053-2064.

[17] Campbell C M, Fulton J P, Mcdonald T P, Wood C W, Zech W C, Srivastava P. Spinner-disc technology to enhance the application of poultry litter. Applied Engineering in Agriculture, 2010; 26(5): 759-767.

[18] Su N, Xu T S, Song L T, Wang R J, Wei Y Y. Variable rate fertilization system with adjustable active feed-roll length. Int J Agric \& Biol Eng, 2015; 8(4): 19-26

[19] Dong X Q, Song J N, Zhang J K, Kang X J, Wang J C. Working performance and experiment on granular fertilizer spreader with cone disk. Transactions of the CSAE, 2013; 29(19): 33-40. (in Chinese)

[20] Lv J Q, Shang Q Q, Yang Y, Li Z H, Li J C, Liu Z Y. Performance analysis and experiment on granular fertilizer spreader with cone disc. Transactions of the CSAE, 2016; 32(11): 16-24. (in Chinese)

[21] Chen S F, Zhang S P, Sun X Z, Li Y M. Design and experiment of self-propelled high-ground-clearance spreader for paddy variable-rate fertilization. Transactions of the CSAE, 2012; 28(11): 16-21. (in Chinese)

[22] Hu Y G, Yang Y C, Xiao H R, Li P P. Simulation and parameter optimization of centrifugal fertilizer spreader for tea plants. Transactions of the CSAM, 2016; 47(5): 77-82.

[23] Shi Y Y, Chen M, Wang X C, Odhiambo M O, Zhang Y N, Ding W M. Analysis and experiment of fertilizing performance for precision fertilizer applicator in rice and wheat. Transactions of the CSAM, 2017; 48(7): 97-103. (in Chinese)

[24] ISO5690-2-1984. Equipment for distributing fertilizers-test methods-Part 2: Fertilizer distributors in lines.

[25] ASAE S341.2. Procedure for measuring distribution uniform and calibrating granular broadcast spreaders.

[26] Yu H, Liu B Q, Ying J J, Hu W G. One-dimension image edge detection method based on sigmoidal function fitting. Infrared Technology, 2014; 36(10): 816-819. 